\title{
Taking population stratification into account by local permutations in rare- variant association studies on small samples
}

\author{
J. Mullaert ${ }^{1,2,3}$, M. Bouaziz ${ }^{3,4}$, Y. Seeleuthner ${ }^{3,4}$, B. Bigio ${ }^{5}$, J-L. Casanova ${ }^{3,4,5,6,7}$, A. Alcais ${ }^{3,4}$, \\ L. Abel ${ }^{3,4,5, \$}$, A. Cobat ${ }^{3,4, \$, *}$
}

1. Université de Paris, IAME, INSERM, F-75018 Paris, France

2. AP-HP, Hôpital Bichat, DEBRC, F-75018 Paris, France

3. Laboratory of Human Genetics of Infectious Diseases, Necker Branch, INSERM

UMR1163, Paris, France, EU

4. Université de Paris, Imagine Institute, 75015 Paris, France, EU.

5. St. Giles Laboratory of Human Genetics of Infectious Diseases, Rockefeller Branch, The Rockefeller University, New York, USA.

6. Howard Hughes Medical Institute, New-York, NY, USA

7. Pediatric Hematology and Immunology Unit, Necker Hospital for Sick Children, 75015

Paris, France, EU.

$\$$. These authors contributed equally to this work

* Corresponding author: aurelie.cobat@inserm.fr

Running title: LocPerm for rare variant association studies 
bioRxiv preprint doi: https://doi.org/10.1101/2020.01.29.924977; this version posted January 30, 2020. The copyright holder for this preprint (which was not certified by peer review) is the author/funder, who has granted bioRxiv a license to display the preprint in perpetuity. It is made available under aCC-BY-NC-ND 4.0 International license.

Keywords: rare-variant association study, population stratification, permutation, principal component analysis 


\section{Abstract}

2 Many methods for rare variant association studies require permutations to assess the significance of

3 tests. Standard permutations assume that all individuals are exchangeable and do not take population

$4 \quad$ stratification (PS), a known confounding factor in genetic studies, into account. We propose a novel

5 strategy, LocPerm, in which individuals are permuted only with their closest ancestry-based

6 neighbors. We performed a simulation study, focusing on small samples, to evaluate and compare

7 LocPerm with standard permutations and classical adjustment on first principal components. Under

8 the null hypothesis, LocPerm was the only method providing an acceptable type I error, regardless of

9 sample size and level of stratification. The power of LocPerm was similar to that of standard

10 permutation in the absence of PS, and remained stable in different PS scenarios. We conclude that

11 LocPerm is a method of choice for taking PS and/or small sample size into account in rare variant

12 association studies. 
14

\section{Introduction}

Population stratification (PS) is a classic confounding factor in genetic association studies of common variants (1). It also affects association studies involving rare variants in the context of next-generation sequencing (NGS) analyses (2-4). Principal component analysis (PCA) is the most widely used approach to correction for stratification. The use of principal components (PCs) computed from common variants as covariates in a regression framework to test for association has been widely investigated $(1,5,6)$. This strategy provides a satisfactory correction in a number of settings, but is subject to several limitations, particularly in cases of complex population structure $(4,7)$. In addition, the regression framework implicitly assumes an asymptotic distribution of the test statistics, which is rarely achieved when sample size is small (8), and few studies of PC-based correction in this context have been published (9).

Permutation methods (particularly the derivation of an empiric distribution by the random permutation of phenotype labels) are classically used in strategies for deriving $p$-values from a test statistic with a probability distribution that is unknown or from which it is difficult to sample (10). However, this approach assumes that all individuals are equally interchangeable under the null hypothesis, an assumption that is not valid in the presence of PS (11). When ancestry is known, it is reasonable to ensure that permutations result exclusively in the exchange of individuals of the same ancestry, but this information is rarely available in practice. We investigated the impact of PS on association studies based on NGS data in the context of limited sample sizes, a situation frequently observed in rare disorders. We propose a new method, LocPerm, based on population-adapted permutation and taking into account the genetic distance between individuals. We describe a detailed analysis of its properties with respect to PC adjustment and standard permutation. 


\section{Materials and methods}

We propose a new approach, LocPerm, in which permutation is restricted such that each individual can be exchanged only with one of its nearest neighbors in terms of a PC-based genetic distance (Supplementary note). Here, we focused on a binary phenotype and the cohort allelic sum test (CAST) approach (12) implemented in a logistic regression framework, using the likelihood ratio test (LRT) statistic. The LocPerm p-value can be calculated by either the usual full empiric $(F E)$ approach (in which the $p$-value is equal to the number of permutation samples with a test statistic as extreme as that observed, divided by the total number of permutation samples), or a semi-empiric (SE) approach. In the SE approach, a limited number of permuted statistics are used to estimate the mean $(\mathrm{m})$ and standard deviation $(\sigma)$ of the test statistic under the null hypothesis, and the $p$-value is calculated from the $\mathrm{N}\left(\mathrm{m}, \sigma^{2}\right)$ distribution (Supplementary note).

We performed a simulation study based on real NGS data, to assess the type I error and power of the LocPerm procedure in the context of small sample sizes. We compared LocPerm to the asymptotic CAST approach with (CAST-3PC) and without (CAST) inclusion of the three principal components (PCs) in the regression model, and to standard permutations applied to CAST. We extracted 1,523 individuals - 745 of Southern European ancestry, 651 of Central European ancestry and 127 of Northern European ancestry (eFigure1) - from our in-house HGID (Human Genetic of Infectious Diseases) whole-exome sequencing dataset and the public 1000 Genomes Phase 3 whole-genome sequencing dataset (Supplementary note). Under the null hypothesis, cases and controls were randomly drawn from the source population according to three PS scenarios (absence of PS, intermediate and extreme stratification, supplementary note). For power analysis, we selected one gene with a cumulative frequency of rare variants of $6.2 \%$, for which we simulated a binary phenotype in the source population, assuming a relative risk of the disease of 4 for individuals carrying at least one rare variant. We then conducted a sensitivity analysis to investigate the effect on the type I error of the number of neighbors in the LocPerm procedure. 
The results of the simulation study under the null hypothesis $\left(\mathrm{H}_{0}\right)$ for the three stratification scenarios and various sample sizes are shown in Table 1 (for $\alpha=0.01$ ) and eTable 1 (for $\alpha=0.05$ ). In the absence of PS, methods based on test statistics following an asymptotic distribution (CAST and CAST-3PC) had inflated type I errors for small sample sizes. Stronger inflation was observed for CAST-3PC than for CAST (e.g. type I error $=0.0124$ vs. 0.0114 at $\alpha=0.01$ for samples of 30 cases and 180 controls). Methods based on permutation (standard and LocPerm) gave correct type I errors. In the presence of PS, the strongest type I error inflation was observed for CAST. The addition of the first three PCs to the model took PS into account only partially. Inflated type I errors were also observed for standard permutations in the presence of PS. Type I error inflation increased with the degree of PS and with sample size for CAST and standard permutation, whereas small sample size appeared to be the main source of inflation for CAST-3PC. The LocPerm procedures ( $F E$ and $S E$ ) provided type I errors close to the expected $\alpha$ threshold across all sample sizes and PS scenarios, despite being slightly conservative in the presence of extreme stratification, particularly for LocPerm-SE.

We further investigated the sensitivity of the LocPerm procedure to the number of neighbors under $\mathrm{H}_{0}$ (Figure 2). With an $\alpha$ threshold of 0.01 , the type I error of the LocPerm procedure remained stable over a wide range of numbers of neighbors (from 20 to 170 for a total sample of 210 individuals), and the use of 30 neighbors appeared to be a reasonable choice. The results of the simulation study under the alternative hypothesis are shown in Figure $\mathbf{1}$ for methods providing a non-inflated type I error rate (i.e. standard permutation in the absence of stratification and LocPerm-SE and LocPerm-FE with and without stratification). In the absence of stratification, a similar power was achieved for standard permutation, LocPerm-FE and LocPerm-SE (43\%, $42 \%$ and $42 \%$ at $\alpha=0.01$ for standard permutation, LocPerm-FE and LocPerm-SE, respectively). In the presence of extreme stratification, the power of LocPerm-FE was well conserved, whereas that of LocPerm-SE decreased slightly, consistent with its conservative type I error rate in this scenario. 


\section{Discussion}

88 The inclusion of the first few PCs in the association model is a popular strategy for taking population

89 structure into account. However, it is suitable only for methods implemented in a regression

90 framework and requires large sample sizes. We found that, in small samples, inclusion of the first

91 three PCs in CAST failed to control the type I error in the presence of PS. In situations in which

92 permutations were required, the LocPerm procedure proposed here took PS into account effectively,

93 with no significant power loss relative to other methods in the absence of PS. The SE approximation

94 performed well in all scenarios, being only slightly conservative in the context of extreme PS and reducing the computational cost by a factor 10 relative to the $F E$ approach. We did not include adaptive permutation (13), in which the number of permutation samples decreases as the observed $p$ value increases, in our comparison. However, we would expect the $S E$ approximation to be faster than adaptive permutation because it requires only 500 permutation samples, whatever the observed $p$ value.

A permutation approach handling PS was proposed in a previous study (14). The odds of disease conditional on covariates were estimated under a null model of no genetic association, and individual phenotypes were resampled, using these disease probabilities as individual weights, to obtain permuted data with a similar PS. However, subsequent studies showed that this procedure was less efficient than regular PC correction for dealing with fine-scale population structure (15). We show here that LocPerm, which uses the first 10 PCs weighted by their eigenvalues to compute a genetic distance matrix, handles complex and extreme PS more effectively than the standard PC-based correction approach, particularly in the context of small sample size. We focused here on binary traits and the CAST approach, but it should be straightforward to extend the LocPerm approach to quantitative traits and other rare variant association tests, particularly for adaptive burden tests requiring permutations. 
114 Acknowledgment

115 We thank both branches of the Laboratory of Human Genetics of Infectious Diseases for

116 helpful

117 discussions and support.

119 Conflict of interest

120 All authors declare no conflict of interest related to this work.

123 The Laboratory of Human Genetics of Infectious Diseases was supported in part by grants 124 from the French National Agency for Research (ANR) under the "Investissement d'avenir" 125 program (grant number ANR-10-IAHU-01), the TBPATHGEN project (ANR-14-CE14-0007126 01), the MYCOPARADOX project (ANR-16-CE12-0023-01), the Integrative Biology of 127 Emerging Infectious Diseases Laboratory of Excellence (grant number ANR-10-LABX-62128 IBEID), the St. Giles Foundation, the National Center for Research Resources and the 129 National Center for Advancing Sciences (NCATS), and the Rockefeller University. 
1. Price AL, Zaitlen NA, Reich D, Patterson N. New approaches to population stratification in genome-wide association studies. Nat Rev Genet. 2010 Jul;11(7):459-63.

2. O'Connor TD, Kiezun A, Bamshad M, Rich SS, Smith JD, Turner E, et al. Fine-scale patterns of population stratification confound rare variant association tests. PloS One. 2013;8(7):e65834.

3. Tintle N, Aschard H, Hu I, Nock N, Wang H, Pugh E. Inflated type I error rates when using aggregation methods to analyze rare variants in the 1000 Genomes Project exon sequencing data in unrelated individuals: summary results from Group 7 at Genetic Analysis Workshop 17. Genet Epidemiol. 2011;35 Suppl 1:S56-60.

4. Mathieson I, McVean G. Differential confounding of rare and common variants in spatially structured populations. Nat Genet. 2012 Feb 5;44(3):243-6.

5. Price AL, Patterson NJ, Plenge RM, Weinblatt ME, Shadick NA, Reich D. Principal components analysis corrects for stratification in genome-wide association studies. Nat Genet. 2006 Aug;38(8):904-9.

6. Zhang Y, Guan W, Pan W. Adjustment for population stratification via principal components in association analysis of rare variants. Genet Epidemiol. 2013 Jan;37(1):99-109.

7. Liu Q, Nicolae DL, Chen LS. Marbled inflation from population structure in gene-based association studies with rare variants. Genet Epidemiol. 2013 Apr;37(3):286-92.

8. Bigdeli TB, Neale BM, Neale MC. Statistical properties of single-marker tests for rare variants. Twin Res Hum Genet Off J Int Soc Twin Stud. 2014 Jun;17(3):143-50.

9. Jiang Y, Epstein MP, Conneely KN. Assessing the Impact of Population Stratification on Association Studies of Rare Variation. Hum Hered. 2013;76(1):28-35.

10. Good P. Permutation Tests: A Practical Guide to Resampling Methods for Testing Hypotheses. New York: Springer-Verlag; 1994. (Springer Series in Statistics).

11. Good P. Extensions Of The Concept Of Exchangeability And Their Applications. J Mod Appl Stat Methods. 2002 Nov 1;1(2).

12. Morgenthaler S, Thilly WG. A strategy to discover genes that carry multi-allelic or mono-allelic risk for common diseases: a cohort allelic sums test (CAST). Mutat Res. 2007 Feb 3;615(12):28-56.

13. Che R, Jack JR, Motsinger-Reif AA, Brown CC. An adaptive permutation approach for genomewide association study: evaluation and recommendations for use. BioData Min. 2014;7:9.

14. Epstein MP, Duncan R, Jiang Y, Conneely KN, Allen AS, Satten GA. A Permutation Procedure to Correct for Confounders in Case-Control Studies, Including Tests of Rare Variation. Am J Hum Genet. 2012 Aug 10;91(2):215-23.

15. Persyn E, Redon R, Bellanger L, Dina C. The impact of a fine-scale population stratification on rare variant association test results. PLOS ONE. 2018 déc;13(12):e0207677. 


\section{Figure legends}

170 Figure 1: Power at 1\% significance level for different PS scenarios, permutation procedures and

171 number of cases and controls in the sample

173 Figure 2: Influence of the number of neighbors for the generation of local permutation (x axis) on

174 type I error (y axis) for the scenario with 30 cases and 180 controls.

175 The situation with 210 neighbors corresponds to standard permutation. 
Table 1. Type I error rates of the different approaches and stratification scenarios at a nominal alpha level of $1 \%$. Type I error rates above the upper bound of the $95 \%$ prediction interval in bold.

\begin{tabular}{|c|c|c|c|c|c|c|c|c|}
\hline Stratification & $\mathrm{N}$ cases & Ncontrols & $\mathrm{N}$ genes* & CAST & CAST-3PC & Std. Perm & LocPerm FE & LocPerm SE \\
\hline \multirow{9}{*}{ Absence } & 30 & 60 & 136932 & 1.09 & 1.32 & 0.97 & 0.96 & 1.06 \\
\hline & 30 & 120 & 186513 & 1.2 & 1.37 & 0.99 & 0.97 & 0.98 \\
\hline & 30 & 180 & 210287 & 1.14 & 1.24 & 0.96 & 0.98 & 0.94 \\
\hline & 60 & 60 & 167621 & 1.06 & 1.25 & 1 & 1 & 1.02 \\
\hline & 60 & 120 & 200883 & 1.08 & 1.2 & 0.98 & 0.99 & 1.02 \\
\hline & 60 & 180 & 217518 & 1.14 & 1.26 & 0.96 & 0.99 & 1.03 \\
\hline & 120 & 120 & 217319 & 1.05 & 1.16 & 1 & 1.02 & 1.04 \\
\hline & 120 & 180 & 227650 & 1.02 & 1.11 & 0.97 & 1 & 1 \\
\hline & 381 & 1142 & 265365 & 1.02 & 1.04 & 1 & 0.98 & 0.97 \\
\hline \multirow{9}{*}{ Intermediate } & 30 & 60 & 135896 & 1.52 & 1.43 & 1.3 & 0.99 & 0.99 \\
\hline & 30 & 120 & 187282 & 1.62 & 1.41 & 1.33 & 1.01 & 0.93 \\
\hline & 30 & 180 & 210510 & 1.48 & 1.28 & 1.29 & 0.94 & 0.84 \\
\hline & 60 & 60 & 166912 & 1.58 & 1.17 & 1.45 & 0.97 & 0.92 \\
\hline & 60 & 120 & 200578 & 1.74 & 1.24 & 1.58 & 0.98 & 0.94 \\
\hline & 60 & 180 & 217720 & 1.87 & 1.23 & 1.61 & 0.92 & 0.89 \\
\hline & 120 & 120 & 218007 & 2 & 1.12 & 1.91 & 0.98 & 0.9 \\
\hline & 120 & 180 & 228317 & 2.17 & 1.08 & 2.05 & 0.93 & 0.85 \\
\hline & 381 & 1142 & 265365 & 2.09 & 1.13 & 2.04 & 1.03 & 0.9 \\
\hline \multirow{6}{*}{ Extreme } & 30 & 60 & 135054 & 1.57 & 1.53 & 1.37 & 0.87 & 0.74 \\
\hline & 30 & 120 & 187267 & 1.76 & 1.63 & 1.47 & 0.92 & 0.78 \\
\hline & 30 & 180 & 210373 & 1.68 & 1.54 & 1.47 & 0.98 & 0.81 \\
\hline & 60 & 60 & 167433 & 1.74 & 1.2 & 1.64 & 0.81 & 0.63 \\
\hline & 60 & 120 & 201217 & 1.97 & 1.35 & 1.81 & 0.9 & 0.75 \\
\hline & 60 & 180 & 218030 & 2.21 & 1.41 & 1.9 & 0.87 & 0.77 \\
\hline
\end{tabular}




\begin{tabular}{lccccccc}
120 & 120 & 218063 & $\mathbf{2 . 4 6}$ & $\mathbf{1 . 0 9}$ & $\mathbf{2 . 3 2}$ & 0.88 & 0.71 \\
120 & 180 & 228643 & $\mathbf{2 . 7 3}$ & $\mathbf{1 . 2}$ & $\mathbf{2 . 5 9}$ & 0.94 & 0.79 \\
381 & 1142 & 265365 & $\mathbf{3 . 5 9}$ & $\mathbf{1 . 4}$ & $\mathbf{3 . 5 2}$ & 0.91 & 0.74 \\
\hline
\end{tabular}

$* \mathrm{~N}$ protein coding genes with at least 10 carriers of rare variants over 15 replicates 


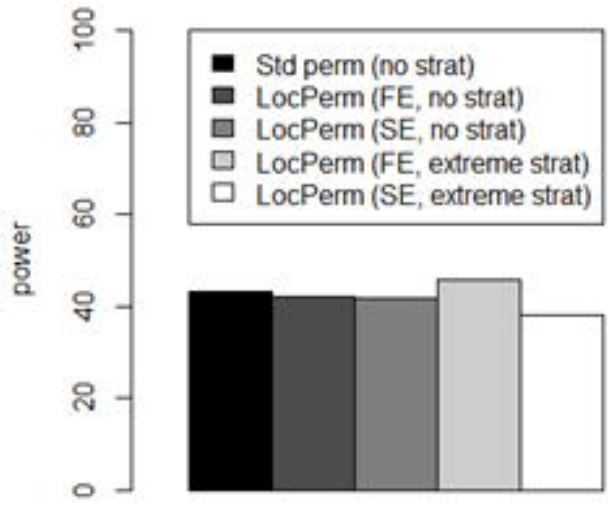

$30 / 120$

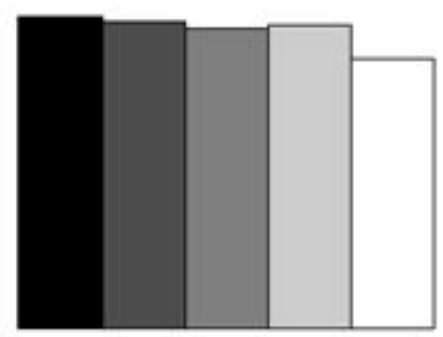

$60 / 120$

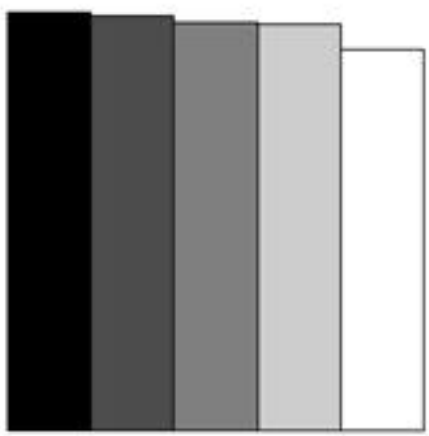

$120 / 180$ 


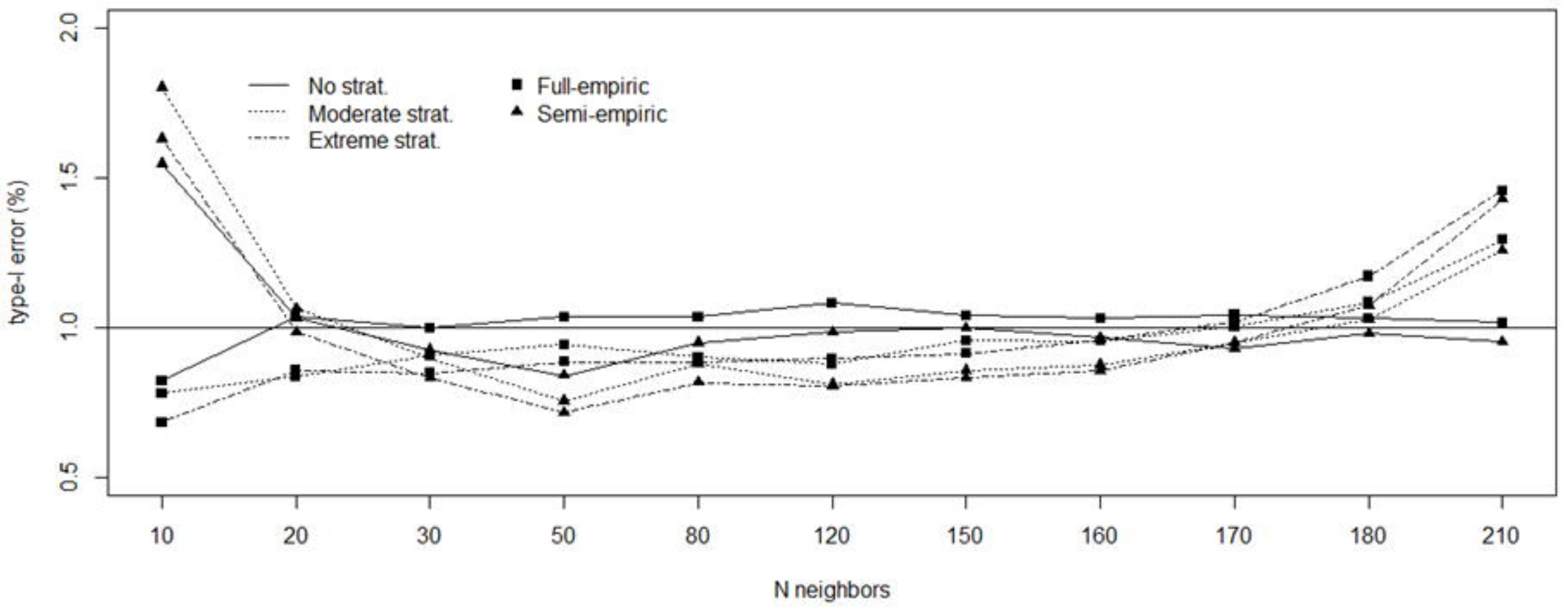

